\title{
ON THE RECONSTRUCTION CONJECTURE FOR SEPARABLE GRAPHS
}

\author{
V. KRISHNAMOORTHY ${ }^{\dagger}$ and K. R. PARTHASARATHY
}

(Received 9 August 1979; revised 26 June 1980)

Communicated by J. N. Crossley

\begin{abstract}
Some sufficient conditions for the reconstructability of separable graphs are given proceeding along the lines suggested by Bondy, Greenwell and Hemminger. It is shown that the structure and automorphism group of a central block plays an important role in the reconstruction.
\end{abstract}

1980 Mathematics subject classification (Amer. Math. Soc.): 05 C 60.

\section{Introduction}

\subsection{Notation and Terminology}

We consider only finite, unoriented, ordinary graphs and follow Harary (1969) for basic terminology and use the following additional terminology.

The maximal subgraph without pendant vertices of a graph $G$ is called the pruned graph of $G$. The block or cutpoint corresponding to the unique central point of the block-cutpoint-tree (bc $(G)$ ) of $G$ is the autocenter of $G$ denoted by $C(G)$ or $C$. The autocenter of the pruned graph is the pruned center $P$ of $G$. Let $v \in V(P)$. If $P$ is a block the maximal connected subgraph of $G$ whose intersection with $P$ is $v$ is the branch of $P$ at $v$. If $P(=v)$ is a cutpoint, then any maximal connected subgraph of $G$ which contains $v$ as a noncutpoint is a branch at $v$. Let $B$ be a block of $G$ and $X$ be a block or cutpoint of $G$. The block distance $D(X, B)$ is the number of blocks between $X$ and $B$, including $B$ and excluding $X$ if $X$ is a block. This is $k$ if the distance between the corresponding

CCopyright Australian Mathematical Society 1981

${ }^{\dagger}$ Research supported by CSIR, India, while the author V. Krishnamoorthy was at Indian Institute of Technology, Madras. 
points of $\mathrm{bc}(G)$ is $2 k$ or $2 k-1$. The block length of a branch at $X$ is defined as $\max D(X, B)$ where $B$ is any block in that branch. A branch at $C$ of maximum length, say $r$, is called a radial branch and $r$ is called the block radius of $G$. A block of $G$ is called a peripheral block if it corresponds to a peripheral point (end of a diameter) of $\mathrm{bc}(G)$. Figure 1 may be helpful in understanding these concepts.

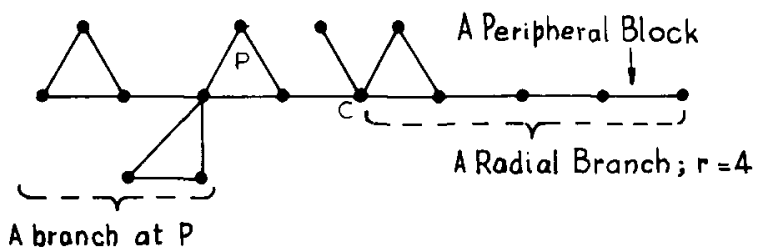

FIGURE 1

By a branch in this paper we always mean a branch at the pruned center $P$.

Let

$$
\begin{aligned}
V_{1} & =\{u \in V \mid \operatorname{deg} u=1\}, \\
\mathcal{G} & =\{G-u \mid u \in V(G)\}, \\
\mathcal{S}_{1} & =\{G-u \mid \operatorname{deg} u=1\} \text { and } \\
\mathcal{G}_{\bar{P}} & =\{G-u \mid u \notin V(P)\},
\end{aligned}
$$

where the multiplicities of the $G-u$ 's are also taken into account.

$G$ is said to be reconstructable (or 1-reconstructable or $\bar{P}_{\text {-reconstructable) }}$ if $G$ is reconstructable from the collection $\mathcal{G}$ (or $\mathcal{G}_{1}$ or $\mathcal{G}_{\bar{P}}$, respectively). Clearly $\mathcal{G} \supseteq \mathcal{G}_{\bar{P}} \supseteq \mathcal{G}_{1}$. Thus, 1 -reconstructability $\Rightarrow \bar{P}$-reconstructability $\Rightarrow$ reconstructability.

\subsection{Review and Scope}

The reconstruction conjecture states that every graph $G$ is reconstructable. This has been settled in the affirmative for a few classes of graphs, in particular for separable graphs without pendant vertices (Bondy (1969)). Bondy's conjecture that $G$ is 1-reconstructable if $\left|V_{1}\right|$ is sufficiently large was disproved in Bryant (1971). However, 1-reconstructability of some classes of separable graphs with pendant vertices was established in Greenwell and Hemminger (1969), making crucial use of the notion of pruned center. In this paper we first show that not all graphs are $\bar{P}$-reconstructable and then give some sufficient conditions for the $\bar{P}$-reconstructability of graphs.

This shows that to settle the reconstruction conjecture for separable graphs eventually one will have to consider cards from the deck $\mathcal{G}_{P}=\{G-u \mid u \in$ $V(P)\}$. One attempt to settle the conjecture may be by establishing the reconstructability of graphs with various mutually exclusive and exhaustive assumptions on the nature of $P$. 


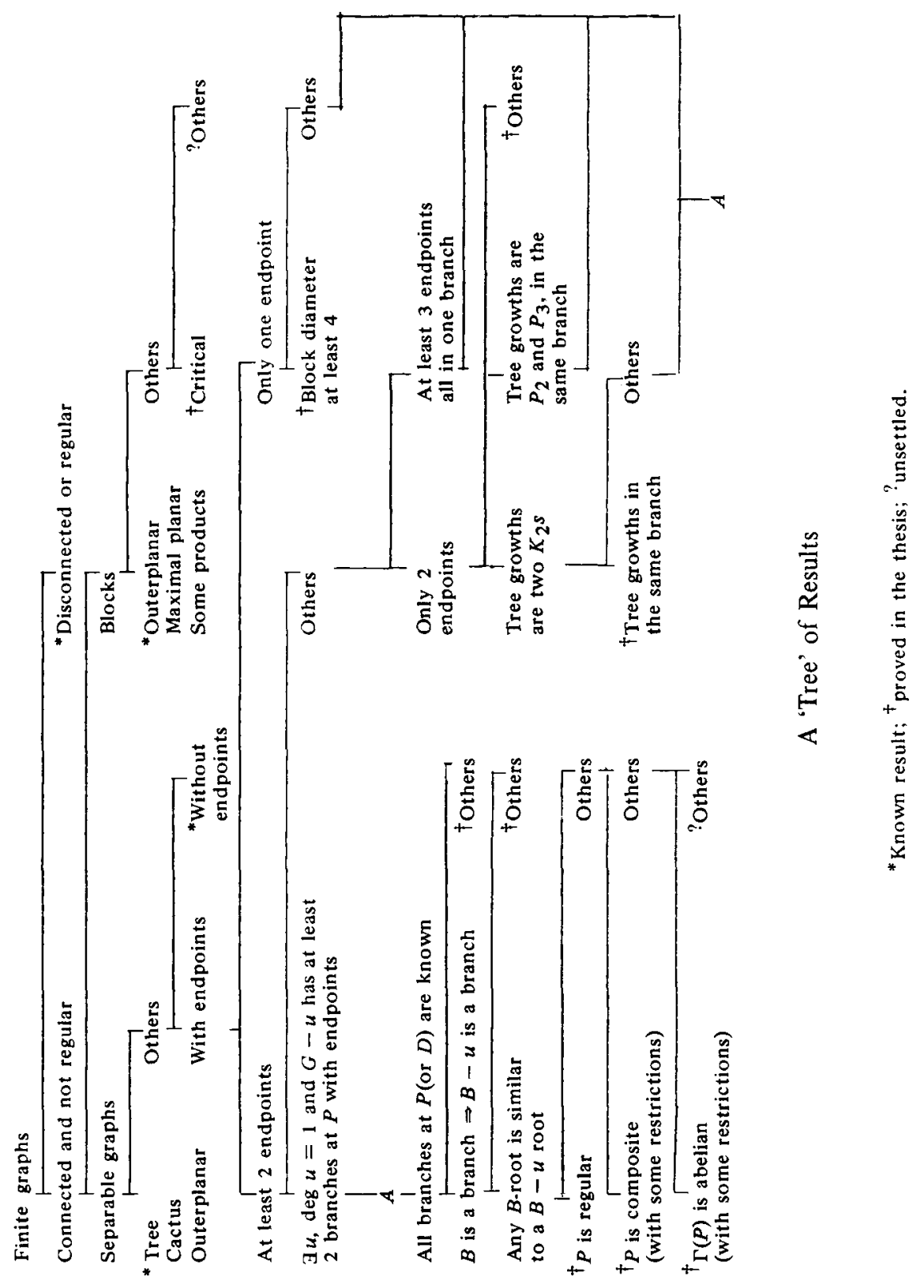


In this paper we show that $G$ is reconstructable if $P$ is regular and $\bar{P}$-reconstructable if $P$ has an abelian automorphism group. In Krishnamoorthy (1976), one of us has established the reconstructability of a graph whose $P$ is a composite block (that is, $P$ is expressible as the Cartesian product of graphs). The 'tree' of results in that thesis is given here for an over all view of the Reconstruction Conjecture with reference to the present work.

\section{A counter example}

In the counter example constructed in Bryant (1971) to show that there are graphs which are not 1-reconstructable, $P$ is a block and all the nontrivial branches are isomorphic to $K_{2}$. Clearly these graphs are also non $\bar{P}_{\text {-reconstructa- }}$ ble. The following steps explain the construction of some graphs which are not $\bar{P}$-reconstructable, and in which the branches are not restricted to be $K_{2}$.

Step 1: Let $\left\{H_{1}, H_{2}, \ldots, H_{m}\right\}$ be a set of distinct nonisomorphic branches (connected rooted graphs) such that if $u$ is any noncutpoint (excluding the root) of $H_{i}$, then $H_{i}-u$ is some $H_{j}$, where $1 \leqslant i, j<m$.

Step 2: Consider a block $H$, such that $\left\{v_{1}, \ldots, v_{m}\right\} \subseteq V(H)$ and the automorphism group of $H$ when restricted to $\left\{v_{1}, \ldots, v_{m}\right\}$ gives $A_{m}$, the alternating group on $m$ letters. Existence of such a graph is proved in Bouwer (1969) and Krishnamoorthy and Parthasarathy (1978).

Step 3: Identifying the root of $H_{i}$ with $v_{i}$, for $1 \leqslant i<m$, we get $G$. Interchange the positions of any two branches to get $G^{\prime}$, non-isomorphic to $G$. $H_{i}$ can be chosen such that $H$ is the pruned center of $G$.

It can be easily seen that the collection $\mathcal{G}_{\bar{P}}$ is the same as the corresponding collection for $G^{\prime}$ and hence $G$ is not $\bar{P}$-reconstructable.

Note 2.1: We can adjust the set $\left\{H_{i}\right\}$ in such a way as to include any given set of branches.

Note 2.2: The counter example can be modified so that the branches occur at different similarity classes of $V(P)$. When we talk of points of $P$ similar to each other we consider $P$ as a graph.

\section{3. $\bar{P}$-reconstructability}

The above counter example leads to the natural question: When is $G \bar{P}$-reconstructable?

We first assume that there exists a pendant vertex $v$ such that $G-v$ has at least two branches with pendant vertices (Assumption I). This gives us all the branches 
of $G$ (at $P$ ) (see Greenwell and Hemminger (1969), p. 105), and hence if $P$ is a cutpoint $G$ is obviously $\bar{P}$-reconstructable. Henceforth let $P$ be a block. Once we know all the branches and $P$, we can find whether $C$ is a cutpoint or not and whether $C$ intersects $P$. This is found by noting that in whatever way the branches are attached to different points of $P, D(C, P)$ is going to be the same.

Theorem 3.1. If $C \cap P \neq C$, then $G$ is 1-reconstructable.

Proof. If $u$ is a pendant vertex of $G$, it is obvious that $C(G-u)$ will be the same as $C(G)$ or $C(G-u)$ will be a cutpoint on $C(G)$ if $C(G)$ is a block and it will be a block containing $C(G)$ if $C(G)$ is a cutpoint. Since $C \cap P \neq C$ and $P$ is a block, in any $G-u \in \mathcal{G}_{1}, C(G-u) \neq P$. Hence in any $G-u \in \mathcal{G}_{1}$, we can find $P(G-u)(=P)$ and fix the branch containing $C(G-u)$ and $C(G)$. Choosing a $G-u \in \mathcal{G}_{1}$ such that the branch at $P$ containing $C(G-u)$ is not a branch of $G$, we can reconstruct $G$, by replacing this branch by the suitable branch of $G$.

Henceforth we assume that $P \cap C=C$.

THEOREM 3.2. If there is a branch $B$ containing a noncutpoint $u$ such that $B-u$ is not a branch of $G$, then $G$ is $\bar{P}$-reconstructable.

Proof. Case 1. $C(G)=P$. In the collection of branches of $G$, replacing one $B$ by $B-u$, we can find $r(G-u)$ and determine whether $C(G-u)$ is a block or a cutpoint.

Case la. Suppose $r(G-u)=r(G)$ and $C(G-u)$ is a block. Then $C(G-u)$ is the same as $P$. Find a $G^{\prime} \in \mathcal{G}$ with $r\left(G^{\prime}\right)=r(G)$ and $C\left(G^{\prime}\right)$ as a block isomorphic to $P$. Moreover choose $G^{\prime}$ such that a branch $B-u$ appears instead of a $B$. $G$ is reconstructed from this $G^{\prime}$ by replacing the branch $B-u$ by $B$.

Case lb. $r(G-u)=r(G)$ and $C(G-u)$ is a cutpoint. This implies that $u$ is the only pendant vertex in the only peripheral block in $B$ and there exists only one more branch with peripheral blocks and $P(G-u)=P(G)$ and $C(G-u)$ is a cutpoint on $P(G)$. Considering such a graph $G^{\prime} \in \mathcal{G}$, since we can find out $P(G-u)=P(G)$ in that $G^{\prime}$, we can reconstruct $G$ by replacing the branch $B-u$ by $B$.

Case 1c. $r(G-u)>r(G)$. As before we know whether $C(G-u)$ is a cutpoint or a block. Choose a $G^{\prime} \in \mathcal{G}$ which has a block isomorphic to $P$, having all the branches of $G$ except one $B$ replaced by $B-u$. If the choice of such a block isomorphic to $P$ in $G^{\prime}$ is unique, then $G$ is reconstructed in the usual way. Suppose there are at least two choices for $P$ in $G^{\prime}$. It can be easily checked (Krishnamoorthy (1976)) that the other choices for $P$ are only among the blocks 
in between $P$ and $B_{v}$ (where $G^{\prime}=G-v$ and $B_{v}$ is the block containing the point $v$ ) or the blocks in $B_{v}-v$. Hence the existence of the second choice for $P$ in $G^{\prime}$ implies that the number of points in $B$ is more than the total number of points in all the other branches and $P$. Hence if $B$ contains a pendant vertex $w$, then clearly $B-w$ is also not a branch and in this case we will be in one of the previous cases. So, let there be no pendant vertex in $B$.

Since there are at least two pendant vertices in the other branches and as these branches appear in $B-v$ (by the second choice for $P$ ), and since $B$ does not have pendant vertices, these pendant vertices of $B-v$ have come from the points of degree 2 in $B_{v}$ and they are adjacent to $v$ in $G$. Now we can choose a point $w$ of degree 2 (adjacent to at most one point of degree 2) in $B_{v}$ which gives only one end vertex in $B-w$. In this case, there will be only one choice for $P$ in $G-w$ and hence $G$ is reconstructed.

Case 2. $C(G)$ is a cutpoint on $P$. The proof is not much different from that of Case 1 and is omitted.

This completes the proof.

Henceforth we assume that if $E$ is a branch of $G$, then so also is any $E-w$, $w \in V(E)$ (Assumption II). This leads to the following observations.

COROLlaRY 3.3. $P$ is the block with maximum number of points in $G$.

Note 3.4. The deck $\mathcal{G}_{\bar{P}}$ can be recognized in $\mathcal{G}$.

Note 3.5. In any $G^{\prime} \in \mathcal{S}_{\bar{P}}, P$ can be identified.

Note 3.6. In any $G-u$, where $u \in V(P)$ and $P-u$ is a block, $P-u$ can be identified.

Theorem 3.2 is sharpened as follows.

THeOREM 3.7. Suppose there is a branch $B$ of $G$, rooted at $v \in V(P)$ and $a$ noncutpoint $u$ of $B$ such that no branch isomorphic to $B-u$ is rooted at a point similar to $v$ in $P$. Then $G$ is $\bar{P}$-reconstructable.

Proof. Let $S_{1}, S_{2}, \ldots, S_{k}$ be the similarity classes in $P$ where $P$ is considered as a graph. These are known, as $P$ is known. Note that there will be nontrivial $\left(\neq K_{1}\right)$ branches attached to at least two $S_{i}$ 's, because of Theorem 3.2 and the assumption following it. First we find the branches at each $S_{i}$ separately.

Consider a $G^{\prime} \in \mathcal{G}_{\bar{P}}$ which contains maximum number of points at the branches in an $S_{i}$. Clearly this gives all the branches at $S_{i}$, as the deleted point has come from a branch attached to some $S_{j}$, where $i \neq j$.

Let $B$ be a branch attached to a point in $S_{1}$, say, such that $B-u$ is not a branch in any point in $S_{1}$. Now considering a $G^{\prime} \in \mathcal{G}_{\bar{P}}$ where at $S_{1}$, one $B-u$ 
appears instead of a $B$, we can reconstruct $G$. (Note that by Note 3.4, $P$ is identified in any $G^{\prime} \in \mathcal{G}_{\bar{P}}$.) This completes the proof.

Henceforth we assume: If $B$ is a branch with a noncutpoint $u$, at a point $v$ of $P$, then there is a branch isomorphic to $B-u$ at a point similar to $v$ in $P$ (Assumption III).

\section{Reconstructability}

Theorem 3.7 highly restricts the separable graphs that are left out to be considered for reconstruction. It is of interest to know under what conditions on $P, G$ will be $\bar{P}$-reconstructable or reconstructable.

The following example shows that $P$ is regular does not imply the $\bar{P}$-reconstructability of $G$.
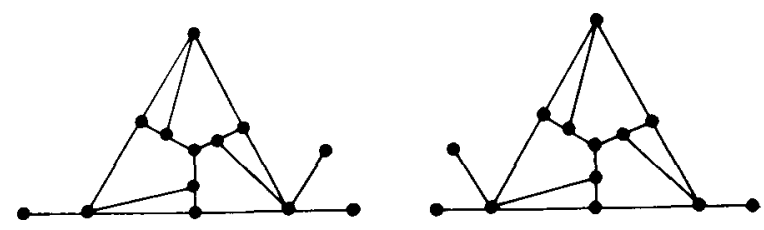

FIGURE 2

However we have

THEOREM 4.1. If $P$ is regular then $G$ is reconstructable.

Proof. Case 1. $P$ is regular of degree $>3$. Then $P$ is a noncritical block (Behzad and Chartrand (1971), Theorem 2.10). So, there is a point $u$ in $P$ such that $P-u$ is a block. Now consider a $G^{\prime} \in \mathcal{G}$ such that there is no block isomorphic to $P$ and there is a block isomorphic to $P-u$. Since $P$ is regular, it can be reconstructed from the $P-u$ in $G^{\prime}$ and the missing branch if any, can be attached to the new vertex added to $P-u$, to get $G$.

Case 2. $P$ is a cycle. In this case $P$ is the largest cycle in $G$ and proceeding exactly as in the case of unicyclic graphs (Manvel (1969)) $G$ is reconstructed.

The proof is complete.

\section{Abelian groups}

We first show that under certain conditions, the only counter examples one can have to $\bar{P}$-reconstructability are the ones described in Section 2 . We know 
that the group $A_{n}$ plays a vital role in these examples and in an attempt to avoid this group, we consider abelian groups in this section.

Let $\Gamma$ be the restriction of $\Gamma(P)$ to $S$ where $S=A \cup B$ and $A=\{x \in$ $V(P) \mid x$ is a nontrivial root in $P\}$ and $B=\{y \in V(P) \mid y$ is similar (in $P$ ) to some $x \in A\}$. Let $G$ satisfy assumption I. Hence we know $P$ and all the branches of $G$. Suppose $G$ is not $\bar{P}$-reconstructable. This implies that Assumptions II and III are also satisfied and if $H$ is any graph with $\mathcal{S}_{\bar{P}}=\mathcal{K}_{\overline{P(H)}}$, then clearly $P(H)=P$, the branches of $H$ are the same as those of $G$ and the only difference between $G$ and $H$ is in the attachment of the branches to $P$.

THEOREM 5.1. Let $G$ be non $\bar{P}$-reconstructable and $\mathcal{G}_{\bar{P}}=\mathcal{K}_{\bar{P}}$. Let $S$ defined above be a similarity class. If every branch (including the trivial one) occurs only once at the points of $S$ then $\Gamma$ is the alternating group $A_{n}$, where $|S|=n$.

Proof. By assumption II, if $B$ is a branch of $G$, so also is any $B-u, u$ a noncutpoint of $B$. Let $B_{i}$ denote the branch at the point $i$ of $P$. Let

$$
S=\{1, \ldots, n\} \quad \text { where }\left|V\left(B_{i}\right)\right|<\left|V\left(B_{j}\right)\right| \quad \text { if } i<j .
$$

Hence $B_{1}=K_{1}, B_{2}=K_{2}$ and $\left|V\left(B_{3}\right)\right|=3$. Consider the $G^{\prime} \in \mathcal{G}_{\bar{p}}$ where one $K_{2}$-branch (that is, a branch isomorphic to $K_{2}$ ) is missing. In this $G^{\prime}$, there are two points in $S$ with trivial branches and since, by assumption, $G$ is not $\bar{P}$-reconstructable, by attaching a $K_{2}$ to the point 1 , we must get $H$ of the

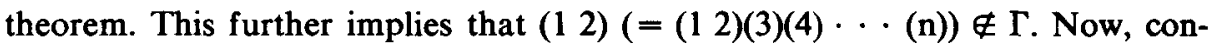
sider a $G^{\prime}$ in which $B_{3}$ is missing and instead a $K_{2}$ appears at 3 . Since the corresponding $H^{\prime}$ is isomorphic to this $G^{\prime}$, we get $\left(\begin{array}{lll}1 & 2 & 3\end{array}\right) \in \Gamma$. Hence $\Gamma$ gives $A_{3}$ on $\{1,2,3\}$ when restricted as identity on $\{4,5, \ldots, n\}$ pointwise.

Let the following statement be the basis for induction. " $\Gamma$ gives $A_{r}$ on $\{1,2, \ldots, r\}$, when it is restricted as identity on $\{r+1, \ldots, n\}$ pointwise, where $3<r<n$."

We show that $\Gamma$ when restricted as identity on $\{r+2, \ldots, n\}$ pointwise, gives $A_{r+1}$ on $\{1,2, \ldots, r+1\}$. Let $u$ be a noncutpoint of $B_{r+1}$. Let $B_{r+1}-u$ $\cong B_{k}$ where $2 \leqslant k \leqslant r$. Then $G-u \cong H-u$ implies that $(12)(k r+1) \in \Gamma$. Using this, it can be easily shown that $\Gamma$ contains all the even permutations on $\{1,2, \ldots, r+1\}$ when restricted as identity on $\{r+2, \ldots, n\}$ pointwise. Since this group cannot be the symmetric group $S_{r+1}$ (since it does not contain (12)) it is $A_{r+1}$. Thus, the proof is complete by induction.

Now, we assume that $\Gamma$ is abelian. (This is slightly more general than assuming that $\Gamma(P)$ is abelian).

If $\Gamma$ is transitive (on $S$ ), that is when $S$ is a similarity class of $P$, we have $|\Gamma|=|S|$ and there is only one element in $\Gamma$ taking a point $u \in S$ to a point 
$v \in S$ (Wielandt (1964), Proposition 4.4). Hence labeling any arbitrary point of $S$, say $v$, as $e$, the identity element of $\Gamma$, we can uniquely label the other points of $S$ with the other elements of $\Gamma$ as follows. If $\alpha(v)=w$, then label $w$ as $\alpha$.

If $\Gamma$ is intransitive, that is when $S$ is a union of (more than one) similarity classes, restricting $\Gamma$ to each of the similarity classes, we can proceed just as above.

THEOREM 5.2. Let $\Gamma$ be transitive on $S$. Let there be at least 3 branches in $G$ with at least one of them different from $K_{2}$. Then $G$ is $\bar{P}$-reconstructable.

Proof. Let $B$ be a branch such that $|V(B)|>3$.

Case 1. Suppose there is only one $B$-branch in $G$. We can assume that $B$ has maximum number of points among all such branches appearing only once. From a $G^{\prime}$ where one $K_{2}$-branch is missing, we can find the positions (in terms of labeling) of the other branches with respect to the $B$-root (that is, root of a $B$-branch) at $e$. If there is a branch $D$, apart from the missing $K_{2}$, such that $D-u \neq B$, then from the corresponding $G-u$ where one $D$-branch is missing, we can find the position of the missing $K_{2}$ in $G^{\prime}$ and hence $G$ is reconstructed.

Suppose there is only one $K_{2}$-branch and for every other branch $D \neq B$, $D-u \cong B$. This implies that $|V(B)|=3$ and $|V(D)|=4$ and the number of $D$-branches is at least two. Now, consider all the graphs (in $\mathcal{G}_{\bar{P}}$ ) in which two $B$-branches appear. In each of these graphs label the unique $K_{2}$-root as $e$ and the $B$-roots accordingly. Only the labels of the $B$-roots are common in all these graphs. This indicates the position of the missing $D$-branch in any such graph and hence $G$ is reconstructed.

Case 2. Every non $K_{2}$-branch appears at least twice. Let $B$ be such a branch with maximum number of points. Consider a $G^{\prime}$ where one $K_{2}$-branch is missing. Let $\left\{e, \alpha_{1}, \ldots, \alpha_{k}\right\}$ be the labels of the $B$-roots starting with some $B$-root as $e$.

Subcase 2.a. Suppose starting with some other $B$-root, say $\alpha_{i}$, we get the same labels for the $B$-roots. This implies that

$$
\left\{e, \alpha_{1}, \ldots, \alpha_{k}\right\}=\left\{\alpha_{i}^{-1}, \alpha_{i}^{-1} \alpha_{1}, \ldots, \alpha_{i}^{-1} \alpha_{k}\right\} .
$$

That is, in any labelling of $S$ if there is a $B$-branch at $\beta$, then there are $B$-branches at $\alpha_{i} \beta$ and $\alpha_{i}^{-1} \beta$ also. Hence in any $G^{\prime}$ where one $B$-branch is missing we can easily find the position of the missing $B$-branch and hence $G$ is reconstructed.

Subcase 2.b. Suppose starting with each $B$-root as $e$, we let different sets of labels for the $B$-roots. Let $\left\{e, \alpha_{1}, \ldots, \alpha_{k}\right\}$ be the labels of $B$-roots starting from a certain $B$-root, say $v$. If all the $B$-branches are present in a $G^{\prime}$, then we can distinguish the point $v$ in $G^{\prime}$. 
Suppose there is a branch $D$ different from $B$ and $K_{2}$. Since $|V(B)|$ is maximum, any $D-u \neq B$. Now from a $G^{\prime}$ where one $K_{2}$-branch is missing, find the labels of the $D$-roots with the point $v$ as $e$. Now from a $G^{\prime \prime}$ where one $D$-branch is missing, $G$ is easily reconstructed.

Suppose the branches different from $B$ are all isomorphic to $K_{2}$. Hence $|V(B)|=3$ (by Assumption II). If there are at least two $K_{2}$-branches, then comparing two graphs where one $K_{2}$-branch is missing in each, we can reconstruct $G$ (as we can fix $v$ in both these graphs). So, let there be only one $K_{2}$-branch in $G$. Suppose $\left\{\beta_{1}, \ldots, \beta_{k}\right\}$ are the labels of the $B$-roots where the $K_{2}$-root is $e$ (we do not know these labels now). Consider a $G^{\prime}$ in which there are two $K_{2}$-branches. So there are two choices for fixing the $K_{2}$-branch. One choice gives the labels $\left\{\beta_{1}, \ldots, \beta_{k}\right\}$ for the $B$-roots and the other choice gives $\left\{\beta_{i}^{-1} \beta_{1}, \ldots, \beta_{i}^{-1} \beta_{k}, \beta_{i}^{-1}\right\}-\{e\}$ where $1 \leqslant i \leqslant k$. Considering some other similar $G^{\prime \prime}$, we can get

$$
\left\{\beta_{1}, \ldots, \beta_{k}\right\} \text { and }\left\{\beta_{j}^{-1} \beta_{1}, \ldots, \beta_{j}^{-1} \beta_{k}, \beta_{j}^{-1}\right\}-\{e\},
$$

where $j \neq i$. If

$$
\left\{\beta_{i}^{-1} \beta_{1}, \ldots, \beta_{i}^{-1} \beta_{k}, \beta_{i}^{-1}\right\} \neq\left\{\beta_{j}^{-1} \beta_{1}, \ldots, \beta_{j}^{-1} \beta_{k}, \beta_{j}^{-1}\right\}
$$

then we can distinguish $\left\{\beta_{1}, \ldots, \beta_{k}\right\}$ and using this in the $G^{\prime}$ considered, $G$ is reconstructed. Otherwise,

$$
\left\{\beta_{i}^{-1}, \beta_{i}^{-1} \beta_{1}, \ldots, \beta_{i}^{-1} \beta_{k}\right\}=\left\{\beta_{j}^{-1}, \beta_{j}^{-1} \beta_{1}, \ldots, \beta_{j}^{-1} \beta_{k}\right\},
$$

where $1 \leqslant i, j \leqslant k$.

This implies

$$
\left\{\beta_{i}^{-1}, \beta_{i}^{-1} \beta_{1}, \ldots, \beta_{i}^{-1} \beta_{k}\right\}=\left\{e, \beta_{1}^{-1}, \beta_{2}^{-1}, \ldots, \beta_{k}^{-1}\right\} .
$$

Multiplying all the elements by $\beta_{i}$, we get

$$
\left\{e, \beta_{1}, \ldots, \beta_{k}\right\}=\left\{\beta_{i}, \beta_{i} \beta_{1}^{-1}, \beta_{i} \beta_{2}^{-1}, \ldots, \beta_{i} \beta_{k}^{-1}\right\} .
$$

Hence every $\beta_{i} \beta_{j}^{-1} \in\left\{e, \beta_{1}, \ldots, \beta_{k}\right\}$ (where $1 \leqslant i, j \leqslant k$ ) and

$$
\left\{\beta_{i}^{-1}, \beta_{i}^{-1} \beta_{1}, \ldots, \beta_{i}^{-1} \beta_{k}\right\}=\left\{e, \beta_{1}, \ldots, \beta_{k}\right\} \text {. }
$$

This shows $\left\{e, \beta_{1}, \ldots, \beta_{k}\right\}$ is a subgroup of $\Gamma$.

Hence starting from any $B$-root as $e$, we will get the labels of the $B$-roots as $\left\{e, \beta_{1}, \ldots, \beta_{k}\right\}-\left\{\beta_{i}\right\}$ for some $i$, and the $K_{2}$-branch will be at $\beta_{i}$. Now $G$ is easily reconstructed from the $G^{\prime}$ where the $K_{2}$-branch is missing.

THEOREM 5.3. Let $\Gamma$ be intransitive. Let there be at least 3 branches with at least one of them different from $K_{2}$. Then $G$ is $\bar{P}$-reconstructable.

Proof. As we can deal with each of the similarity classes of $S$ separately, by the previous theorem it is enough to consider the case where each of the 
similarity classes having a non $K_{2}$-branch has just two nontrivial branches. This implies that any (non-trivial) non $K_{2}$-branch has just 3 points. Let $S_{1}$ be a similarity class of $S$ with the non $K_{2}$-branch $B$. From a $G^{\prime}$ where one $K_{2}$-branch is missing from some other similarity class, we can find the label of the $K_{2}$-root at $S_{1}$, with the $B$-root at $S_{1}$ as $e$. Now from the graph in which the $K_{2}$-branch at $S_{1}$ is missing, $G$ is reconstructed. This completes the proof.

The remaining case is when all the branches are isomorphic to $K_{2}$. In this case we prove a theorem under the assumption that $\Gamma$ is cyclic and transitive.

THEOREM 5.4. Let $\Gamma$ be cyclic and transitive. Let all the branches be isomorphic to $K_{2}$. If the number of branches is at least 4 , then $G$ is $\bar{P}$-reconstructable.

Proof. Since $\Gamma$ is cyclic and transitive on $S$, any generator of $\Gamma$ contains a single cycle of length $|S|$. Let $(12 \cdots k)$ be such a generator. In any $P\left(G^{\prime}\right)$, $G^{\prime} \in \mathcal{S}_{\bar{P}}$, we can fix the orientation given by this cycle. We can imagine a directed cycle corresponding to $(12 \cdots k)$ and for our purpose, think that $P$ is replaced by this directed cycle.

(Note that here we cannot proceed as in the case of unicyclic graphs, by deleting the points of the cycle, since the cycle is an imaginary one.)

Two nontrivial roots are said to be consecutive if there is a path joining them in the (imagined) directed cycle such that the other points of the path are trivial roots. A new distance between two consecutive roots is defined as the length of such a path.

Since all the branches are isomorphic to $K_{2}$, it is enough to find the distances between the consecutive roots in the cyclic order. First, we determine these distances without any order. For this, choose a $G^{\prime} \in \mathcal{G}_{\bar{p}}$ which has a maximum distance, say $p$, between two consecutive roots. Since this $p$ is a sum of two adjacent distances of $G$, the other distances of this $G^{\prime}$ are distances in $G$ also. Let $r, s$ be two adjacent distances of $G$ given by $G^{\prime}$. Consider a $G^{\prime \prime} \in \mathcal{G}_{\vec{P}}$ with maximum number of distances equal to $r+s$ and minimum number of distances equal to $r$. Clearly by splitting a distance $r+s$ in $G^{\prime \prime}$ as $r$ and $s$, we get all the distances of $G$.

In any $G^{\prime}$, which has a distance $p$, we can find out which combination of distances (without any order), say $m$ and $n$, has given rise to the distance $p$, by comparing the distances of $G$ and $G^{\prime}$. If $m=n, G$ is easily reconstructed. Hence for definiteness let $m>n$. Let $c_{m n}$ be the number of combinations of adjacent distances $m$ and $n$ in $G$ giving rise to $p$ where the distance $m$ occurs first (in the cyclic order). Let $c_{n m}$ be defined similarly. Let $c=c_{m n}+c_{n m}$. Clearly $c$ is the number of graphs in $\mathcal{G}_{\bar{P}}$ with a distance $p$ appearing, instead of an $m$ and an $n$. 
Of course, we don't know the numbers $c_{m n}$ and $c_{n m}$ now, though we know $c$. Let $c_{m n}^{\prime}, c_{n m}^{\prime}, c^{\prime}$ denote the corresponding numbers for any $G^{\prime} \in \mathcal{G}_{\bar{P}}$ with a distance $p$. These numbers are, of course, known.

Case 1. Suppose $c>1$. Once we know the numbers $c_{m n}$ and $c_{n m}$ we can reconstruct $G$ as follows. Five different cases arise.

(a) $c^{\prime}=c-1 ; c_{m n}^{\prime}=c_{m n}-1 ; c_{n m}^{\prime}=c_{n m}$.

(b) $c^{\prime}=c-1 ; c_{m n}^{\prime}=c_{m n} ; c_{n m}^{\prime}=c_{n m}-1$.

(c) $c^{\prime}=c-3 ; c_{m n}^{\prime}=c_{m n}-1 ; c_{n m}^{\prime}=c_{n m}-2$.

(d) $c^{\prime}=c-3 ; c_{m n}^{\prime}=c_{m n}-2 ; c_{n m}^{\prime}=c_{n m}-1$.

(e) $c^{\prime}=c-2 ; c_{m n}^{\prime}=c_{m n}-1 ; c_{n m}^{\prime}=c_{n m}-1$.

In the first case the distance $p$ in $G^{\prime}$ is replaced by $m$ and then $n$ to get $G$. The second case is similar. In case $c$, the distances in $G^{\prime}$ will be of the form $\ldots n, p, m \ldots$ and they are replaced by $n, m, n, m, \ldots$ to get $G$. In case $d$, the distances $\ldots m, p, n, \ldots$ of $G^{\prime}$ are replaced by $\ldots m, n, m, n, \ldots$ Suppose every $G^{\prime}$ considered is in case $e$. The four possibilities for the consecutive distances with $p$ in the middle are

(3) $\ldots r, n+m, n, \ldots ; r \neq m$ and

(2) $\ldots m, n+m, r, \ldots ; r \neq m, n$

(4) $\ldots n, m+n, r, \ldots ; r \neq m$.

The splitting of $m+n$ is easily found in the first two cases and also in the other cases if $r \neq n$. Suppose $r=n$. Now, consider a $G^{\prime \prime}$ with a distance $p$ and minimum number of $n$ 's occurring consecutively after $p$. In this $G^{\prime \prime}$ clearly $m$ appears first in the splitting of $p$. Hence $G$ is reconstructed.

Now our aim is to find $c_{m n}$ and $c_{n m}$. If there are at least two different combinations of adjacent distances of $G$, such that $m+n=p=x+y$ then from a $G^{\prime}$ where $x$ and $y$ give rise to $p$, we can find $c_{m n}$ and $c_{n m}$. Suppose only the combination $m$ and $n$ gives $p$. If there is a $G^{\prime}$ where $c^{\prime}=c-2$, then $c_{m n}=c_{m n}^{\prime}+1$ and $c_{m n}=c_{n m}^{\prime}+1$. If there is $G^{\prime}$ where $c^{\prime}=c-3$, then $c_{m n}$ and $c_{n m}$ are easily found out from our discussion of the reconstruction of cases $c$ and $d$. So, in every $G^{\prime}$ considered, let $c^{\prime}=c-1$. We can easily find from all these graphs whether $c_{m n}$ or $c_{n m}$ is zero or not. If both are different from zero, a $G^{\prime}$ with maximum $c_{m n}^{\prime}$ gives $c_{m n}$ and hence $c_{n m}$ also. Suppose $c_{m n}=0$. Then in any $G^{\prime}, c_{n m}^{\prime}=c_{n m}-1$. Hence $c_{n m}$ is found.

Case 2. $c=1$. Consider the unique $G_{1}$ with the distance $p$. Let the distances in this be $\ldots r, p, s, t, \ldots$ Suppose $r \neq s$. Then consider a $G^{\prime}$ which does not have the consecutive distances $\boldsymbol{m}$ and $n$ and which has minimum number of distances equal to $m$. In this if the new distance formed is $m+r$ then $m$ occurs first and then $n$, and if the new distance formed is $m+s$, then $m$ occurs after $n$. Suppose $r=s$. Clearly $r \neq m, n$ (since $c=1$ ). Two cases arise depending on $t$. If $t \neq n$, consider a $G^{\prime}$ where there are minimum number of distances equal to $m$, maximum number of distances equal to $m+s$ and the combination $(m, n)$ 
does not occur. If in this $G^{\prime}$ the distance $n$ occurs after a distance $m+s$, then $m$ occurs first and then $n$ in $p$. Otherwise $n$ occurs first and then $m$ in $p$.

Suppose $t=n$. Then $n$ will occur after $m+s$, whether $m$ occurs first or not in $p$. But now, consider a $G^{\prime}$ in which the combination $(m, n)$ does not occur and in which the distances $n$ and $s$ combine to give $n+s$. If there is an $m$ after an $n+s$, in the $G^{\prime}$, clearly $n$ occurs first in the $(m, n)$ combination. Otherwise $n$ occurs first.

This completes the proof.

Note 5.5. Theorem 5.4 is not true when there are only three $K_{2}$-branches. For example, consider $P$ where $S=\{1,2, \ldots, 6\}$ and $\Gamma$ is the cyclic group on 6 elements whose generator is ( 123456 ). By choosing the distances as 1,2 and 3 (in that order) we get $G$. If we chose the distances 1,3 and 2 (in that order) we get $H \approx G$. It is easy to see that $G$ is not $\bar{P}$-reconstructable.

This counterexample can be modified such that the pruned center is a cycle. The $G$ and $H$ for this case are shown in Figure 3.
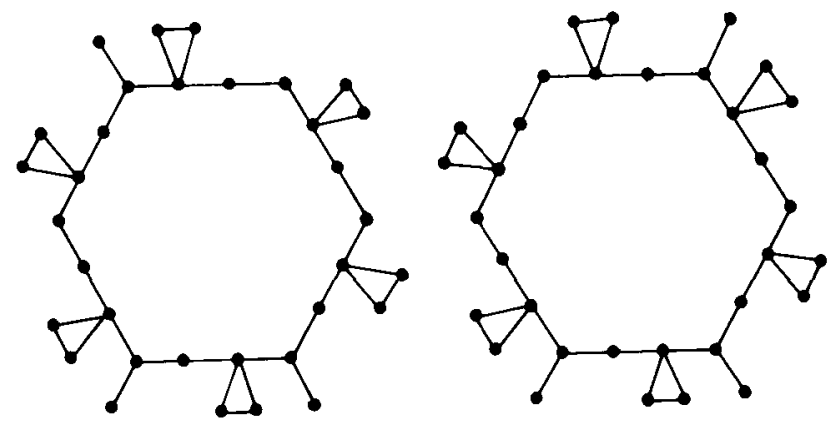

FIGURE 3

This shows that the corresponding subcases of Theorem 4.10 and Corollaries 4.13 and 4.14 in Greenwell and Hemminger (1969) are not true.

\section{References}

M. Behzad and G. Chartrand (1971), Introduction to the theory of graphs (Allyn and Bacon, Boston).

J. A. Bondy (1969), 'On Ulam's conjecture for separable graphs', Pacific J. Math. 31, 281-288.

I. Z. Bouwer (1969), 'Section graphs for finite permutation groups', The many facets of graph theory, edited by G. Chartrand and S. F. Kapoor, pp. 55-61, Lecture Notes in Mathematics 110, Springer Verlag, Berlin.

R. M. Bryant (1971), 'On a conjecture concerning the reconstruction of graphs', J. Combinatorial Theory Ser. B 11, 139-141. 
D. L. Greenwell and R. L. Hemminger (1969), 'Reconstructing graphs', The many facets of graph theory, edited by G. Chartrand and S. F. Kapoor, pp. 91-114, Lecture Notes in Mathematics 110, Springer Verlag, Berlin.

F. Harary (1969), Graph theory (Addison-Wesley, Reading, Mass.).

V. Krishnamoorthy (1976), 'The reconstruction conjecture and some related problems' (Ph. D. thesis, I. I. I. Madras).

V. Krishnamoorthy and K. R. Parthasarathy (1978), 'F-sets in graphs', J. Combinatorial Theory Ser. B 24, 53-60.

B. Manvel (1969), 'Reconstruction of unicyclic graphs', Proof techniques in graph theory, edited by F. Harary, pp. 103-107 (Academic Press, New York).

H. Wielandt (1964), Finite permutation groups (Academic Press, New York).

Faculty of Science

Madras Institute of Technology Campus

Perarignar Anna University of Technology

Madras-44

India
Department of Mathematics Indian Institute of Technology Madras-36 India 